\section{International Scientific Journal Theoretical \& Applied Science}

p-ISSN: 2308-4944 (print) e-ISSN: 2409-0085 (online)

Year: 2017 Issue: $11 \quad$ Volume: 55

Published: 27.11.2017 http://T-Science.org
Oleg Sabinin

$\mathrm{PhD}$ in Computer Science

Peter the Great St. Petersburg Polytechnic University

Saint-Petersburg, Russia

Ekaterina Sheikina

Researcher

Peter the Great St. Petersburg Polytechnic University

Saint-Petersburg, Russia

SECTION 4. Computer science, computer engineering and automation.

\title{
MULTIDIMENSIONAL DATABASES AS A DATA SOURCE OF BUSINESS INTELLIGENCE SYSTEMS
}

\begin{abstract}
The aim of this paper is to compare a relational database, an optimized relational database, OLAP as a model of multidimensional databases and formulate the prerequisites of the usage of MOLAP, ROLAP and HOLAP on the basis of theoretical facts and an implemented experiment in terms of Business Intelligence technologies. The formulated prerequisites refer to data sources' performance, supported amount of data, features of required analysis, available disk space, frequency of reporting and volatility of data. The implemented experiment includes the comparison of average performance of databases, MOLAP, ROLAP and HOLAP with the constant number of rows in a fact table and the increasing complexity of queries, the comparison of average performance of databases and MOLAP with increasing number of rows and observations about average disk space allocated by MOLAP. Furthermore, brief descriptions of Business Intelligence systems, OLAP and its basic forms were given.

Key words: Business Intelligence, databases, MOLAP, ROLAP, HOLAP.

Language: English

Citation: Sabinin O, Sheikina E (2017) MULTIDIMENSIONAL DATABASES AS A DATA SOURCE OF BUSINESS INTELLIGENCE SYSTEMS. ISJ Theoretical \& Applied Science, 11 (55): 175-180.

Soi: http://s-o-i.org/1.1/TAS-11-55-21 Doi: crossef https://dx.doi.org/10.15863/TAS.2017.11.55.21
\end{abstract}

\section{Introduction}

Nowadays the vast majority of companies are interested in their financial stability, increase of their net income and competitive strength. There is a great variety of means which make it possible for enterprises to accomplish the above mentioned purposes. The list of these means includes competently implemented managerial policy, marketing means and the usage of different information technologies represented on the market.

Business Intelligence (BI) systems are one of the most suitable solutions which enable to control the current business state and crucial trends, assess business decisions and spread obtained information. As the parts of Business Intelligence technologies user - friendly interface, well - organized and well visualized dashboards and reports, which can be created in various formats and shared with different devises and embedded alert capabilities play a significant role regarding decision - making and business assessment.

Nevertheless, data sources of Business Intelligence systems also should be taken into account. The quality and elaborateness of generated dashboards and reports, the speed of information processing and the amount of consumed resources depend on the right chosen data source. In connection with this state of affairs the expected volume of data storage, limited resources, necessary degree of analytics represented via dashboards should be taken into consideration before the implementing a data source.

Hence, the goal of this paper is to compare different data sources including relational databases and OLAP cubes regarding their usage in BI technologies. The stated goal will be achieved by conducting an experiment which shows the relation between data sources' performance and the complexity of queries and the volume of a fact table and formulation of prerequisites of the usage of a particular data source.

The paper is organized as follows: brief description of Business Intelligence technologies, features of multidimensional databases including MOLAP, ROLAP and HOLAP, description of the conducted experiment and its results, formulated prerequisites of data sources' usage and conclusions. 


\section{Business Intelligence technologies}

The term of Business Intelligence systems was firstly introduced in the middle of the $20^{\text {th }}$ century. However, the expanded version of this term that was similar to the contemporary conception of BI appeared in the 1990s due to Howard Dresner, analyst of the Gartner Group. The Gartner Group defines Business Intelligence as "an umbrella term that includes the applications, infrastructure and tools, and best practices that enable access to and analysis of information to improve and optimize decisions and performance" [1, p. 12].

In other words, Business Intelligence systems are analytical systems, which unite data obtained from various data sources, process these data in a particular way, which makes it possible to estimate received information, carry out business analysis and make decisions which contribute to a favorable outcome [2, p. 122]. BI systems are generally considered to be software applications that deliver information to decision makers to help maintain business performance [3, p. 2]. Obviously, the core functionality of these systems boils down to data transformation into information and knowledge about the state of business from different angles.

The most frequently obtained benefits from the usage of Business Intelligence systems consist in increasing of degree of business activity control and reduction of costs. The first category of BI advantages is associated with high degree of visualization of current trends of business provided by various analytical capabilities. The above mentioned features contribute to timely decision making, therefore, to efficiency of an established managerial policy.

Moreover, timely creation and sharing of reports, which are able to reflect to the changes of requirements, reduction of the human factor's influence, prediction and alert means are considered as BI benefits. Speaking about the decreasing of costs, it should be noted that BI reduces IT infrastructure costs by eliminating redundant data extraction processes and duplicate data housed in independent data marts across the enterprise [4, $\mathrm{p}$. 97]. However, an empirical study for 50 Finnish companies found most companies do not consider cost or time savings as primary benefit when investing in BI systems [5, p. 185].

\section{Features of multidimensional databases}

Business Intelligence technologies support a great variety of data sources including relational databases, multidimensional databases and external files. However, the aim of this section of the paper is scrutinizing Online Analytical Processing, or OLAP, as a model of multidimensional databases which views data as cubes.

The conception of OLAP was firstly introduced by E. Codd in 1993. Online Analytical Processing systems provide fast answers for queries that aggregate large amounts of detail data to find overall trends [6, p. 40]. The OLAP cubes form the data storage and data organizing layers of a BI system [7, p. 86]. The described conception meets the requirements of FASMI principle, or Fast Analysis of Shared Multidimensional Information.

In spite of the fact that the conception provided by Codd has evolved since the date of its creation, different components of OLAP still play a significant role. The list of the components includes dimensions, hierarchies, measures and facts. Dimensions represent the set of characteristics which determine the orientation of data and the degree of their detailing. The more dimensions a cube has, the more detailed data are. For instance, "Products" can be considered as a dimension. The characteristics included in a particular dimension form hierarchies, or level - organized objects. In other words, the dimension is organized in hierarchies to enable analysis of the measures at various levels of detail. The hierarchies of dimension define the structures for aggregating measures [8, p. 3]. Hence, "category of a product", "type of a product" and "name of a product" are the levels of the hierarchy regarding dimension "Products".

In connection with the nature of OLAP systems, they include different calculations, which work with aggregated data stored in a cube. These calculations, or measures, can be divided into two types: numeric values and expressions based on formulas. Gross margin, sales, COGS, operating income, net income are considered as the examples of measures. Finally, facts represent the data located on the intersection of cells and which should be analyzed.

Multidimensional databases in terms of Online Analytical Processing exist in three basic forms, which are applicable to contemporary Business Intelligence systems. These forms are MOLAP, or Multidimensional OLAP, ROLAP, or Relational OLAP, and HOLAP, or Hybrid OLAP.

MOLAP is distinguished from other OLAP's forms with the following important characteristic: aggregated data and basic data are stored in a cube in an optimized format. In connection with this characteristic MOLAP cubes are able to execute complex queries efficiently. Nevertheless, because of redundant storage of data MOLAP cubes are limited with the amount of data which should be processed and analyzed and require more disk space in comparison with other models. However, the percentage of required space will be shown in the next section. Furthermore, the latency is high, because every change regarding data in a database requires full processing of a cube [9, p. 10].

According to the model of ROLAP, data are stored in a relational format keeping the slicing concept of OLAP. Hence, the amount of data which can be analyzed is limited with the capacity of a 
database and the complexity of queries is determined by SQL capabilities. This model is characterized by low latency, because of its storage properties. Nevertheless, the performance of ROLAP is lower than the performance of MOLAP, especially, when it is necessary to process huge amounts of data in a detailed way.

HOLAP model represents the combination of benefits of the above mentioned models with the purpose of minimization of their drawbacks. Thus, general data are stored in relational database and aggregated data are stored in a cube. The performance of this model is lower than the performance of MOLAP, but higher than the performance of ROLAP. This tendency is explained by the fact of storing data in a database.

\section{Conducted experiment and its results}

The experiment was implemented in three steps. The first step, or preparatory step, was conducted for data sources' creation. The second step describes dependency between execution time of a query to a particular data sources and the degree of the query's complexity. The third step describes relation between number of rows in a fact table and execution time regarding a data source. The above mentioned steps are described in detailed way in next paragraphs. It should be noted that all steps were performed several times to take into account uniform and not uniform distributions of data in the database.
Moreover, optimization of the database including creation of additional indexes, statistics collection and partitioning of a fact table took place to estimate the performance on each step.

The first step consists in creation of a relational database based on a star scheme. Star scheme was chosen on the basis of its performance characteristics which distinguish it from snowflake scheme. In this model, each group of dimensions are placed in a dimension table, the facts are placed in fact table [10, p. 11]. A fact table consists of 10 million rows, and it is connected with four dimensions whose number of rows is limited with 1000 rows. Four - dimensional MOLAP, HOLAP and ROLAP was created on the basis of data stored in the database.

The second step provides information about the relation between a data source and its ability to work with different kinds of queries including simple and complex queries. The complexity of queries was determined by calculated measures and the degree of grouping. All queries were chosen on the assumption of their implementation in Business Intelligence applications. The Figure 1 and Figure 2 show dependencies between average execution time in milliseconds regarding the relational database, the optimized database, MOLAP, ROLAP and HOLAP and increasing complexity of queries. The Figure 1 represents general results, and the Figure 2 shows results in detailed way in terms of all used data sources but the relational database.

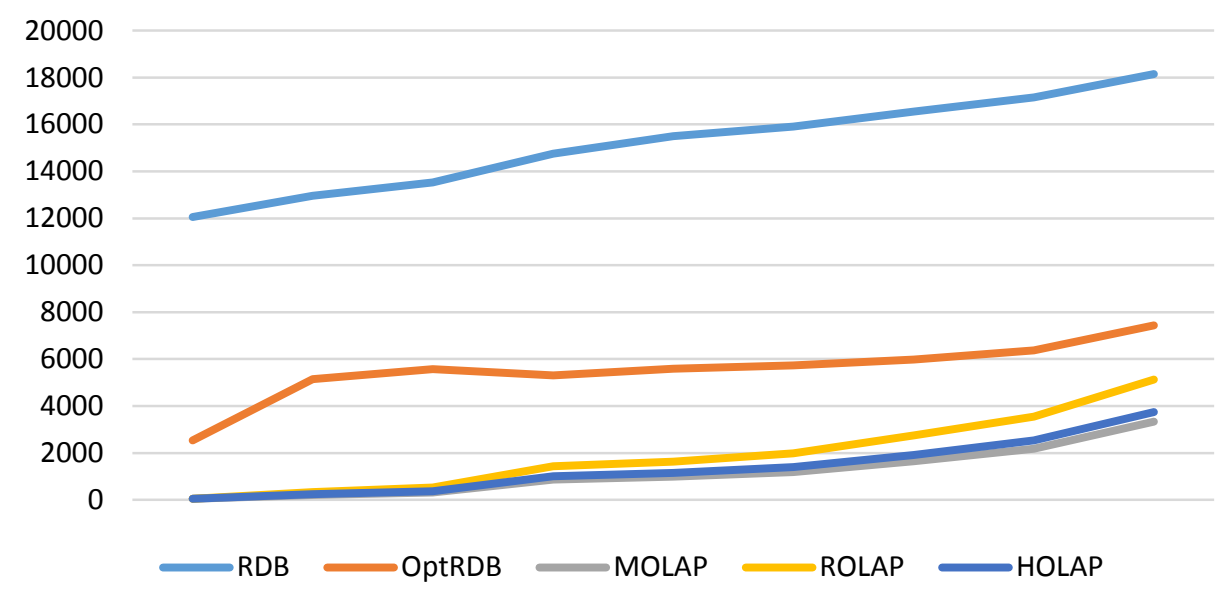

Figure 1 - Comparison of data sources regarding queries' complexity 


\begin{tabular}{l|lrl|l|ll} 
& ISRA (India) & $=\mathbf{1 . 3 4 4}$ & SIS (USA) & $=\mathbf{0 . 9 1 2}$ & ICV (Poland) & $=\mathbf{6 . 6 3 0}$ \\
Impact Factor: & ISI (Dubai, UAE) $=\mathbf{0 . 8 2 9}$ & PUHU (Russia) $=\mathbf{0 . 2 0 7}$ & PIF (India) & $=\mathbf{1 . 9 4 0}$ \\
& GIF (Australia) & $\mathbf{0 . 5 6 4}$ & ESJI (KZ) & $=\mathbf{3 . 8 6 0}$ & IBI (India) & $\mathbf{4 . 2 6 0}$
\end{tabular}

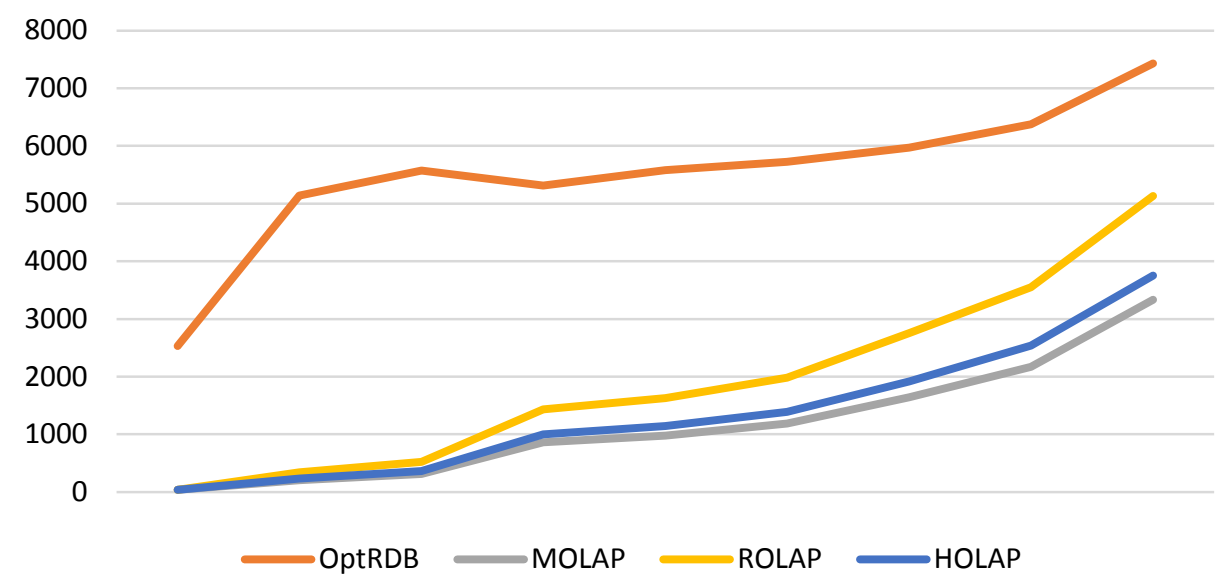

Figure 2 - Detailed comparison of data sources and queries' complexity

As it can be seen from the figures the relational database performance indicators as well as optimized database performance indicators are inferior to other considered data sources. MOLAP shows the shortest average execution time. The difference between MOLAP and HOLAP performance reaches about 17 percent. ROLAP performance is lower than the performance of MOLAP and HOLAP. Nevertheless, ROLAP indicators could be decreased by 60 percent due to the usage of 90 percent aggregation level. However, it should be noted that processing time of the cube will increase in an exponential way.
The third step was implemented to scrutinize the dependency between the volume of the fact table and the execution time in milliseconds of data sources including the relational database, the optimized database and MOLAP. The number of rows stored in the databases vary from 100 rows to 20 million rows. The results of this part of the experiments are shown on Figure 3 and Figure 4. The Figure 3 shows all results of the part of the experiment and the Figure 4 represents results in detailed way in terms of the number of rows in the fact table between 100 and 500000 rows.

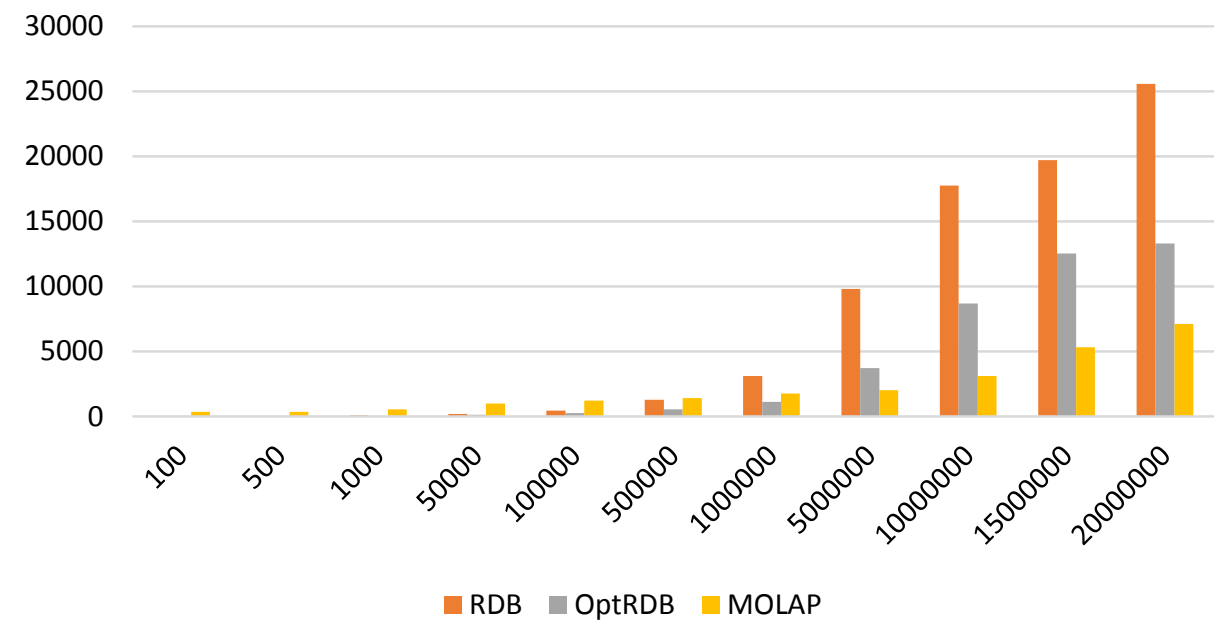

Figure 3 - Comparison of data sources regarding number or rows 


\begin{tabular}{l|lrl|l|ll} 
& ISRA (India) & $=\mathbf{1 . 3 4 4}$ & SIS (USA) & $=\mathbf{0 . 9 1 2}$ & ICV (Poland) & $=\mathbf{6 . 6 3 0}$ \\
Impact Factor: & ISI (Dubai, UAE) $=\mathbf{0 . 8 2 9}$ & PUHU (Russia) $=\mathbf{0 . 2 0 7}$ & PIF (India) & $=\mathbf{1 . 9 4 0}$ \\
& GIF (Australia) & $\mathbf{0 . 5 6 4}$ & ESJI (KZ) & $=\mathbf{3 . 8 6 0}$ & IBI (India) & $\mathbf{4 . 2 6 0}$
\end{tabular}

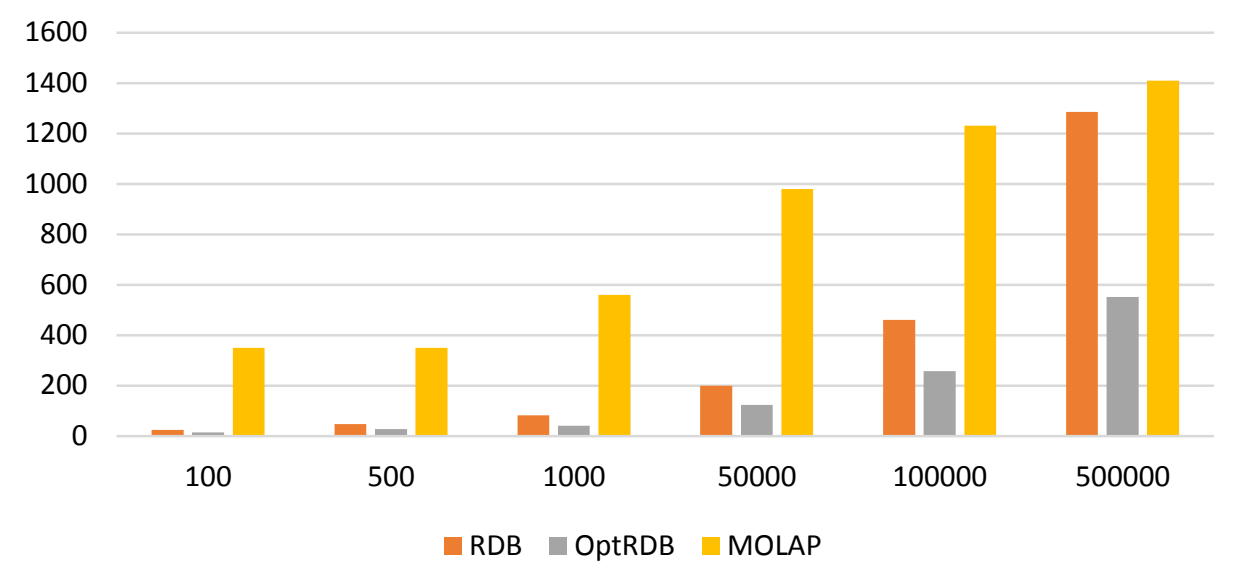

Figure 4 - Detailed comparison of data sources regarding number or rows

During this step of the experiment the comparison of relational database, optimized database and MOLAP was implemented. The results represent that MOLAP cube reaches the highest efficiency when the number of rows in the fact table is higher than 1 million rows. Furthermore, when number of rows is equal to 1 million rows MOLAP cube excels relational database in performance, but it is still inferior to the database improved with optimization methods.

The last but not least fact which should be taken into consideration is disk space allocated by MOLAP cube. High values of space, which exceed the disk space allocated by the relational database in times, were revealed when the number of rows was between 100 and 1000. This tendency can be explained by storage of redundant amount of data in the cube including data about dimensions, measures, hierarchies and metadata. Beginning from 50000 rows the volume of space allocated by MOLAP is less than 31 percent of the disk space allocated by the database. The number of rows from 10 million and 20 million shows the lowest value of space allocated by MOLAP and it is equal to 8 percent.

\section{The prerequisites of the data sources'} usage

On the basis of theoretical knowledge and data obtained due to the conducted experiment it is possible to formulate prerequisites of the usage of described OLAP models and relational databases in terms of Business Intelligence technologies. It should be noted that some prerequisites depend on a company's branch, features of business processes, types of reports and their frequency.

Prerequisites of the MOLAP's usage are the following:

- the necessity of high - detailed reports executed efficiently;

- the necessity of high performance of queries' execution;
- constancy of processed data or the absence of necessity of frequent reports' building;

- in case of low available disk space the amount of data stored in a fact table should be more than 10 million when the amount of data stored in dimensions is relatively low;

- the amount of data stored in a fact table is more than 1 million in comparison with relational databases.

Prerequisites of the ROLAP's usage are the following:

- the absence of necessity of high - detailed reports or high performance of queries' execution;

- necessity of high performance of queries' execution with allowance of high cube's processing time;

- volatility of data stored in a database of the necessity of frequent reports' building;

- limited disk space;

- extremely high amount of data stored in a database;

- necessity of qualitative analysis other than quantitative analysis.

Prerequisites of the HOLAP's usage are the following:

- low but not limited disk space;

- amount of data stored in a database is more than 10 million and the absence of necessity of high-detailed reports and simple reports;

- frequent reporting with qualitative analysis.

Speaking about relational databases, it will be rational to use them in case of the necessity of qualitative analysis and amount of data stored in a fact table should be lower than 1 million rows. Furthermore, it is necessary to use different methods of optimization which are applicable to a star schema. 


\begin{tabular}{|c|c|c|c|c|c|c|}
\hline Impact Factor: & $\begin{array}{l}\text { ISRA (India) } \\
\text { ISI (Dubai, UAF } \\
\text { GIF (Australia) } \\
\text { JIF }\end{array}$ & $\begin{array}{l}=1.344 \\
=0.829 \\
=0.564 \\
=1.500\end{array}$ & $\begin{array}{l}\text { SIS (USA) } \\
\text { PИНЦ (Russia } \\
\text { ESJI (KZ) } \\
\text { SJIF (Morocco }\end{array}$ & $\begin{aligned} &= \mathbf{0 . 9 1 2} \\
&=\mathbf{0 . 2 0 7} \\
&=\mathbf{3 . 8 6 0} \\
&=\mathbf{2 . 0 3 1}\end{aligned}$ & $\begin{array}{l}\text { ICV (Poland) } \\
\text { PIF (India) } \\
\text { IBI (India) }\end{array}$ & $\begin{array}{l}=6.630 \\
=1.940 \\
=4.260\end{array}$ \\
\hline
\end{tabular}

\section{Conclusion}

To sum up, Business Intelligence systems represent the high powerful systems regarding business analytics and business decisions - making. These systems make it possible to create detailed analytical reports and dashboards, identify bottlenecks and determine crucial business trends. The success of implementing of BI systems depends on a data source used by BI. It is crucial to choose the right data source which meets the requirements determined by business features. In connection with this state of affairs the relational database, the optimized relational database and OLAP technologies were scrutinized in the purpose of formulating prerequisites of usage a particular data source. The basic concepts associated with prerequisites refer to data sources' performance, supported amount of data, features of required analysis, available disk space, frequency of reports and volatility of data. The formulated prerequisites are based on theoretical knowledge and the results of the conducted experiment.

\section{References:}

1. Bogdan Nedelcu (2013) Business Intelligence Systems. Database Systems Journal, 4(4), p. 1220.

2. Sabinin OY, Sheikina ES (2017) Automation of making reports in the area of management systems' certification via Oracle Business Intelligence. ISJ Theoretical \& Applied Science, 03(47), p. 121-127.

3. Gregory Richards, William Yeoh, Alain Yee Loong Chong, Ales Popovic (2017) Business Intelligence Effectiveness and Corporate Performance Management: An Empirical Analysis. Journal of Computer Information Systems, p. 1-9.

4. Hugh J. Watson, Barbara H. Wixom (2007) The Current State of Business Intelligence. Computer, Computer, 40(9), p. 96-99.

5. Solomon Negash (2004) Business Intelligence. The communications of the Association for Information Systems, 13(1), p. 177-195.

6. Torben Bach Pedersen, Christian Jensen (2001) Multidimensional Database Technology. Computer, 34(12), p. 40-46.
7. Hussain Al-Aqrabi, Lu Liu, Richard Hill, Nick Antonopoulos (2015) Cloud BI: future of business intelligence in the cloud. Journal of Computer and System Sciences, 81(1), p. 8596.

8. Imen Moalla Ahlem Nabli1 Lotfi Bouzguenda, Mohamed Hammami. (2017) Data warehouse design approaches from social media: review and comparison. Social Network Analysis and Mining, 7(1), p. 1-14.

9. John Palo, Ray, Newman. (2015) Creation and adoption of On-Line Analytical Process (OLAP) into the management decision support system aided by computers. Scholedge International Journal of Business Policy \& Governance, 2(5), p. 8-13.

10. Emany Sidi, Mohamed El Merouani, El Amin Aoulad Abdelouarit (2016) Star Schema Advantages on Data Warehouse: Using Bitmap Index and Partitioned Fact Tables. International Journal of Computer Applications, 134(13), p. 11-13. 